\title{
Extension of Some Results Concerning the Generalized Liénard Equation $\left(^{*}\right)$.
}

\author{
RoLF REISSIG (Bochum)
}

Dedicated to Professor GIovanni Sansone on his 85 th birthday

Summary. $-A$ recent result of Mawhin [7] concerning the existence of forced oscillations for $a$ second order equation of Lienard type with an arbitrary damping term is extended to some cases where the restoring force is not assumed to be sufficiently weak. The results are valid, too, for a certain class of third order equations. They are based on the Leray-Schauder principle. By an analogous argumentation the Rayleigh equation with a quasilinear restoring force, but with an arbitrary damping term, is shown to possess a periodic solution. Again, the result admits an extension to a third order equation.

In the present paper we consider the generalized Liénard differential equation

$$
x^{\prime \prime}+f(x) x^{\prime}+g(x)=e(t) \equiv e(t+T)
$$

[f(x),g(x),e(t) continuous functions of $x$ and $t$, respectively]. We derive a number of conditions for the existence of $T$-periodic solutions which are more general than those of $\mathfrak{J}$. MawhiN [7]. The conditions of Mawhin are characterized by the fact that the function $f(x)$ ean be chosen arbitrarily.

Instead of (1) Mawhin discusses the vector differential equation

$$
\boldsymbol{x}^{\prime \prime}+\frac{d}{d t} \operatorname{grad} F(\boldsymbol{x})+\boldsymbol{g}(\boldsymbol{x})=\boldsymbol{e}(t), \quad \boldsymbol{x} \in R^{n}
$$

some of the following results can also be extended to this equation.

The method of the proof which is applied here is based on the well-known LeraySchauder fixed point theorem. Using a suitable positive constant $\lambda<\omega=2 \pi / T$ we form the equation

$$
x^{\prime \prime}+\lambda^{2} x=\mu\left\{e(t)+\lambda^{2} x-g(x)-f(x) x^{\prime}\right\}, \quad 0<\mu<1,
$$

(*) Entrata in Redazione il 17 luglio 1973. 
and we consider its $T$-periodic solutions. With the aid of the Green function

$$
G(s)=\frac{\sin \lambda s+\sin \lambda(T-s)}{2 \lambda(1-\cos \lambda T)}, 0 \leqslant s \leqslant T,
$$

which is prolongated as a periodic piecewise smooth function, these solutions can be represented as

$$
\begin{aligned}
x(t) & =\mu \int_{0}^{T} G(t-\tau)\left\{e(\tau)+\lambda^{2} x(\tau)-g(x(\tau))\right\} d \tau \\
& -\mu \int_{0}^{T} G^{\prime}(t-\tau) F(x(\tau)) d \tau \\
& =\mu A\{x(t)\} \quad\left(0 \leqq t \leqq T ; F(x)=\int_{0}^{x} f(\xi) d \xi\right) .
\end{aligned}
$$

If they are a priori bounded the existence of at least one periodic solution of equation (3) for each $\mu \in[0,1]$ is ensured by virtue of Leray-Schauder's theorem. Especially equation (1) admits such a solution (case $\mu=1$ ). It should be emphasized that the solutions of integral equation (4) can be interpreted as the fixed points of the transformation $\mu A$ of a suitable Banach space into itself, where $A$ is a bounded, continuous and compact operator.

Notr. - Let the solution $x(t) \equiv x(t+T)$ be expanded into the Fourier series

$$
x(t)=\frac{a_{0}}{2}+\sum_{n=1}^{\infty}\left(a_{n} \cos (n \omega t)+b_{n} \sin (n \omega t)\right),
$$

and, correspondingly, let its first derivative be represented as

$$
x^{\prime}(t)=\omega \sum_{n=1}^{\infty} n\left(b_{n} \cos (n \omega t)-a_{n} \sin (n \omega t)\right) .
$$

Applying Parseval's formula

$$
\|x\|^{2}=\int_{0}^{T} x^{2}(t) d t=T\left\{\frac{a_{0}^{2}}{4}+\frac{1}{2} \sum_{n=1}^{\infty}\left(a_{n}^{2}+b_{n}^{2}\right)\right\},
$$

respectively,

$$
\left\|x^{\prime}\right\|^{2}=\int_{0}^{T} x^{\prime 2}(t) d t=\frac{\omega^{2} T}{2} \sum_{n=1}^{\infty} n^{2}\left(a_{n}^{2}+b_{n}^{2}\right)
$$


we obtain the estimate

$$
\|x\|^{2} \leqslant \frac{1}{T}\left(\int_{0}^{T} x(t) d t\right)^{2}+\frac{\left\|x^{\prime}\right\|^{2}}{\omega^{2}}
$$

Prolongation of the solution as an odd function for $-T \leqq t<0$ yields (in case $x(0)=x(T)=0)$

$$
x(t)=\sum_{n=1}^{\infty} e_{n} \sin \frac{n \omega t}{2}, \quad x^{\prime}(t)=\frac{\omega}{2} \sum_{n=1}^{\infty} n c_{n} \cos \frac{n \omega t}{2} \quad(-T \leqslant t \leqslant+T) .
$$

This time we derive from Parseval's formula that

$$
\|x\|^{2} \leqslant \frac{4}{\omega^{2}}\left\|x^{\prime}\right\|^{2}
$$

Theorem 1. - If

a) $x g(x) \geqq 0(|x| \geqq h>0)$,

b) $\lim _{|x| \rightarrow \infty} \frac{g(x)}{x}=k<\omega^{2}$,

c) $\int_{0}^{p} e(t) d t=0$ in case $k=0$

the equation (1) admits at least one T-periodic solution.

In case $k>0$ we set $\lambda^{2}=k$ and

$$
g(x)=\lambda^{2} x+\gamma(x), \quad \text { where } \lim _{|x| \rightarrow \infty} \frac{\gamma(x)}{x}=0 .
$$

Now we consider equation (3) for a $T$-periodic solution; multiplying it by $x(t)$ and integrating from 0 to $T$ we obtain

$$
-\left\|x^{\prime}\right\|^{2}+\lambda^{2}\|x\|^{2}=\mu \int_{0}^{T} x(t)\{e(t)-\gamma(x(t))\} d t .
$$

Let

$$
\begin{aligned}
& \operatorname{Max}_{[0, T]}|x(t)|=r, \\
& \underset{|x| \leqq r}{\operatorname{Max}}|\gamma(x)|=\Gamma(r)\left[\frac{\Gamma(r)}{r} \rightarrow 0 \text { as } r \rightarrow \infty\right], \\
& \underset{[0, T]}{\operatorname{Max}}|e(t)|=E
\end{aligned}
$$


then we calculate

$$
\left\|x^{\prime}\right\|^{2} \leqq \lambda^{2}\|x\|^{2}+(E+\Gamma) \sqrt{T}\|x\|
$$

Integration of differential equation (3) from 0 to $T$ yields

$$
\begin{gathered}
\lambda^{2} \int_{0}^{T} x(t) d t=\mu \int_{0}^{T}(e(t)-\gamma(x(t))) d t \\
\left|\int_{0}^{T} x(t) d t\right| \leqq \frac{(E+\Gamma) T}{\lambda^{2}} .
\end{gathered}
$$

By means of $(5),(7)$ and (8) we find

$$
\|x\|^{2} \leqq \frac{(E+\Gamma)^{2}}{\lambda^{4}} T+\frac{\lambda^{2}}{\omega^{2}}\|x\|^{2}+\frac{(E+\Gamma) T}{\omega^{2}}\|x\|
$$

since $1>\lambda^{2} / \omega^{2}$ this yields an estimate of the kind

$$
\|x\| \leqq x(E+I)
$$

From (9) we conclude by virtue of (7) that

$$
\left\|x^{\prime}\right\| \leqq x^{\prime}(E+\Gamma)
$$

Note that $x=\varkappa(k, \omega)$ and $x^{\prime}=\chi^{\prime}(k, \omega)$ are system constants. If

$$
x(\tau)=\frac{1}{T} \int_{0}^{T} x(t) d t
$$

for a value $\tau \in[0, T]$ we have on the interval $[\tau, \tau+T]$

$$
\begin{gathered}
x(t)=x(\tau)+\int_{\tau}^{t} x^{\prime}(s) d s, \\
|x(t)| \leqq|x(\tau)|+\sqrt{T^{\prime}}\left\|x^{\prime}\right\| \leqq \lambda^{-8}(E+\Gamma)+x^{\prime} \sqrt{T}(E+\Gamma)=\varkappa_{0}(E+\Gamma),
\end{gathered}
$$

especially

$$
r \leqq \varkappa_{0} E+\varkappa_{0} \Gamma(r) \quad \text { or } 1 \leqq \frac{\varkappa_{0} E}{r}+\varkappa_{0} \frac{\Gamma(r)}{r} \rightarrow 0 \quad\left(r^{\prime} \rightarrow \infty\right)
$$


Consequently

$$
r \leqq R_{0} \quad \text { (independent on } \mu \in(0,1) \text { ) }
$$

In case $k=0$ (already treated by M.AWHTN [7] and, under the restriction $f(x)=c$, by LAzER [5]) we choose an arbitrary $\lambda \in(0, \omega)$ and calculate for a periodic solution $x(t)$ of $(3)$, instead of $(7)$,

$$
\left\|x^{\prime}\right\|^{2} \leqq(1-\mu) \lambda^{2}\|x\|^{2}+(E+\Gamma) \sqrt{T}\|x\|
$$

where

$$
\begin{aligned}
& \operatorname{Max}_{[0, T]}|x(t)|=r, \\
& \operatorname{Max}_{|x| \leqq r}|g(x)|=\Gamma(r) \quad[\Gamma(r) / r \rightarrow 0 \text { as } r \rightarrow \infty] .
\end{aligned}
$$

Integration of equation (3) from 0 to $T$ yields

$$
\int_{0}^{T}\left\{(1-\mu) \lambda^{2} x(t)+\mu g(x(t))\right\} d t=0
$$

since the forcing term $e(t)$ is assumed to possess the mean value zero.

By virtue of $0<\mu<1$ and $x g(x) \geqq 0(|x| \geqq h)$ we obtain for these values $x$

$$
\operatorname{sgn} x\left\{(1-\mu) \lambda^{2} x+\mu g(x)\right\}>0 .
$$

Therefore $|x(t)| \geqq h$ for all $t$ is excluded; there must be a $\tau \in(0, T)$ such that $|x(\tau)|<h$. On the interval $[\tau, \tau+T]$ we have

$$
|x(t)|<h+\sqrt{T}\left\|x^{\prime}\right\|
$$

and, because of (11),

$$
\begin{aligned}
\|x\|^{2} & <2 T h^{2}+2 T^{2}\left\|x^{\prime}\right\|^{2} \\
& \leqq 2 T h^{2}+2 T^{2}\left[\lambda^{2}\|x\|^{2}+(E+\Gamma) \sqrt{T}\|x\|\right] .
\end{aligned}
$$

Choosing $\lambda^{2}<\left(2 T^{2}\right)^{-1}$ we derive an estimate

$$
\|x\| \leqq \varkappa(h+E+\Gamma)
$$

and a similar estimate for $\left\|x^{\prime}\right\|$, due to (11). Now the a priori-boundedness in question is an immediate consequence of (12), compare the previous case.

18 - Annali di Matematica 
THEOREM 2. - If

$$
0<\delta \leqq \frac{g(x)}{x} \leqq h<\frac{\omega^{2}}{4}(|x| \geqq h>0)
$$

then the equation (1) admits at least one T-periodic solution.

We define

$$
\gamma(x)=\left\{\begin{array}{l}
k x-g(x), \quad|x| \geqq h \\
{\left[k-\frac{g(h \operatorname{sgn} x)}{h \operatorname{sgn} x}\right] x, \quad|x| \leqq h}
\end{array}\right.
$$

we have

$$
0 \leqq \frac{\gamma(x)}{x} \leqq k-\delta \quad(x \neq 0)
$$

and

$$
|\varrho(x)|=|k x-g(x)-\gamma(x)| \leqq P \quad(\text { for all } x)
$$

Now we consider equation (3) where again $\lambda^{2}=k$ and $x(t)$ a $T$-periodic solution. We calculate, as above,

$$
\begin{gathered}
-\left\|x^{\prime}\right\|^{2}+k\|x\|^{2}=\mu \int_{0}^{T} x(t)\{e(t)+\gamma(x(t))+\varrho(x(t))\} d t \\
\geqq \mu \int_{0}^{T} x(t)\{e(t)+\varrho(x(t))\} d t \\
\left\|x^{\prime}\right\|^{2} \leqq k\|x\|^{2}+\sqrt{T}(E+P)\|x\|
\end{gathered}
$$

Taking into account that $k<\omega^{2} / 4$ we conclude by virtue of $(6)$ that

$$
\left\|x^{\prime}\right\| \leqq x^{\prime}(E+P)
$$

provided that $x(0)=x(T)=0$. This can be assumed without loss of generality if $x(t) \neq 0$ for all $t$ is exeluded.

An immediate consequence of $(14)$ is

$$
|x(t)|=\left|\int_{0}^{t} x^{\prime}(\tau) d \tau\right| \leqq \sqrt{T}\left\|x^{\prime}\right\| \leqq x(E+P), \quad 0 \leqq t \leqq T .
$$


If, on the contrary, $x(t) \neq 0$ for all $t$ then we obtain by means of integration of equation (3)

$$
\int_{0}^{T}\{k x(t)-\mu \gamma(x(t))\} d t=\mu \int_{0}^{T}\{e(t)+\varrho(x(t))\} d t .
$$

Considering

$$
\delta \leqq(1-\mu) k+\mu \delta \leqq k-\mu \frac{\gamma(x)}{x} \quad(x \neq 0)
$$

we can estimate

$$
\delta \int_{0}^{T}|x(t)| d t \leqq\left|\int_{0}^{T}\{k x(t)-\mu \gamma(x(t))\} d t\right| \leqq(E+P) T
$$

and

$$
\int_{0}^{T}|x(t)| d t=T|x(\tau)| \leqq \frac{(E+P) T}{\delta}
$$

$\tau \in[0, T]$ suitably chosen.

This time from

$$
x(t)=x(\tau)+\int_{\tau}^{t} x^{\prime}(s) d s, \quad \tau \leqq t \leqq \tau+T
$$

it results that

$$
|x(t)| \leqq \frac{E+P}{\delta}+\sqrt{T}\left\|x^{\prime}\right\| \leqq x(E+P)
$$

But the estimate of type (14) which is applied here must be derived from (13) in connexion with (5):

$$
\left\|x^{\prime}\right\|^{2} \leqq 2 k\|x\|^{2}+\frac{T}{2 k}(E+P)^{2} \leqq \frac{2 k}{T} \frac{(E+P)^{2} T^{2}}{\delta^{2}}+\frac{2 k}{\omega^{2}}\left\|x^{\prime}\right\|^{2}+\frac{T}{2 k}(E+P)^{2}\left(1-\frac{2 k}{\omega^{2}}>\frac{1}{2}\right) .
$$

APPENDIX. - If $f(x), g(x), e(t)$ are odd functions and if

$$
0<\delta \leqq \frac{g(x)}{x} \leqq k<\omega^{2} \quad(|x| \geqq h>0),
$$

then equation (1) possesses at least one T-periodic solution.

We note that $-x(-t),-T / 2 \leqq t \leqq 0$, is a solution of equation (3) provided that $x(t), 0 \leqq t \leqq T / 2$, is a solution. 
For we derive from equation (3), setting $g_{\mu}(x)=(1-\mu) \lambda^{2} x+\mu g(x)$ and $\tau=-t$,

$$
\frac{d^{2}}{d \tau^{2}} x(-\tau)-\mu f(x(-\tau)) \frac{d}{d \tau} x(-\tau)+g_{\mu}(x(-\tau))=\mu e(-\tau)
$$

and, multiplying by -1 ,

$$
\frac{d^{2}}{d \tau^{2}}(-x(-\tau))+\mu f(-x(-\tau)) \frac{d}{d \tau}(-x(-\tau))+g_{\mu}(-x(-\tau))=\mu e(\tau),
$$

where we can rewrite $\tau$ into $t$.

Thus, a solution $x(t)$ of $(3)$ on $[0, T / 2]$ fulfilling the boundary condition $x(0)=$ $=x(T / 2)=0$ together with its odd complement $-x(-t)$ is part of a $T$-periodic solution.

The boundary value problem can be solved by means of the Leray-Schauder procedure. All solutions of equation (3) defined on $[0, T / 2]$ and possessing vanishing boundary values satisfy the following integral equation:

$$
\begin{aligned}
x(t)= & -\mu \int_{0}^{\pi / 2} G(t, \tau)\left\{e(\tau)+\lambda^{2} x(\tau)-g(x(\tau))\right\} d \tau \\
& -\mu \int_{0}^{T / 2} G_{\tau}(t, \tau) F(x(\tau)) d \tau
\end{aligned}
$$

where

$$
\left(\lambda \sin \frac{\lambda T}{2}\right) G(t, \tau)= \begin{cases}\sin \lambda\left(\frac{T}{2}-t\right) \sin \lambda \tau, & \tau \leqq t \\ \sin \lambda t \sin \lambda\left(\frac{T}{2}-\tau\right), & t \leqq \tau\end{cases}
$$

A priori-boundedness of all solutions of (16) for $0<\mu<1$ ensures the existence of at least one solution for $\mu=1$.

The proof is similar to that of the preceding Theorem 2. It is based on the Fourier expansions

$$
x(t)=\sum_{n=1}^{\infty} c_{n} \sin (n \omega t), \quad x^{\prime}(t)=\omega \sum_{n=1}^{\infty} n c_{n} \cos (n \omega t)
$$

and on inequality (5) in which the mean value of $x(t)$ is vanishing.

Theorenr 3. - If

a) $x g(\infty) \leqq 0(|\infty| \geqq h)$,

b) $\int_{0}^{T} e(t) d t=0$,

then equation (1) admits at least one T-periodio solution. 
We define

$$
\gamma(x)=\left\{\begin{array}{l}
g(x), \quad|x| \geqq h \\
g(h \operatorname{sgn} x) \frac{|x|}{h}, \quad|x| \leqq h
\end{array}\right.
$$

and we obtain

$$
\gamma(x) x \leqq 0, \quad|g(x)-\gamma(x)| \leqq P \text { for all } x .
$$

With the aid of an arbitrary constant $\lambda>0$ we form the differential equation

$$
x^{\prime \prime}-\lambda^{2} x=\mu\left\{e(t)-\lambda^{2} x-g(x)-f(x) x^{\prime}\right\}, \quad 0<\mu<1,
$$

the $T$-periodie solutions of which can be represented as

$$
\begin{aligned}
x(t) & =\mu \int_{0}^{T} G(t-\tau)\left\{e(\tau)-\lambda^{2} x(\tau)-g(x(\tau))\right\} d \tau \\
& -\mu \int_{0}^{T} G^{\prime}(t-\tau) F(x(\tau)) d \tau,
\end{aligned}
$$

where

$$
\begin{gathered}
G(s)=\frac{\sinh (\lambda s)+\sinh (\lambda(T-s))}{2 \lambda(1-\cosh (\lambda T))}, 0 \leqq s \leqq T, \\
G(s+T) \equiv G(s) \text { for all } s .
\end{gathered}
$$

In order to prove the a priori-boundedness of the solutions of (18) we proceed as above and calculate successively:

$$
\int_{0}^{T}\left\{(1-\mu) \lambda^{2} x(t)-\mu g(x(t))\right\} d t=0,
$$

therefore

$$
\begin{gathered}
|x(\tau)|<h \quad \text { for some } \tau \in(0, T) \\
-\left\|x^{\prime}\right\|^{2}-(1-\mu) \lambda^{2}\|x\|^{2}=\mu \int_{0}^{T} x(t)\{e(t)-\gamma(x(t))-[g(x(t))-\gamma(x(t))]\} d t
\end{gathered}
$$

therefore

$$
\left\|x^{I}\right\|^{2} \leqq \sqrt{T}(E+P)\|x\|
$$

Now we conclude that

$$
|x(t)| \leqq|x(\tau)|+\left|\int^{t} x^{\prime}(s) d s\right|<h+T^{3 / 4} \sqrt{(E+P)\|x\|} \quad \text { for } \tau \leqq t \leqq \tau+T
$$


and

$$
x^{2}(t)<2 h^{2}+2 T^{3 / 2}(E+P)\|x\|
$$

Finally the estimates

$$
\|x\|<\varkappa(h+E+P) \quad \text { and } \quad|x(t)|<\varkappa_{0}(h+E+P)
$$

can be derived where the system constants $x$ and $\varkappa_{0}$ depend on $T$.

APPENDix. Condition a) can be replaced by

$$
x g(x) \leqq 0 \quad\left(|x| \leqq h_{0}=T^{2} E\right) .
$$

It suffices to show that there is no $T$-periodic solution of (17) the maximum norm of which is equal $h_{0}$. If there were such a solution $x(t)$ we would conclude that

$$
(1-\mu) \lambda^{2} x(\tau)-\mu g(x(\tau))=0, \quad \text { i.e. } \quad x(\tau)=0
$$

or some $\tau \in[0, T]$, and by virtue of the preceding result with $h=P=0$ :

$$
\operatorname{Max}|x(t)| \leqq T^{3 / 4} \sqrt{E\|x\|}<\sqrt{h_{0} \operatorname{Max}|x(t)|}
$$

in contradiction to the assumption.

The preceding theorems are applicable to the third order equation

$$
x^{\prime \prime \prime}+a x^{\prime \prime}+f(x) x^{\prime}+g(x)=e(t) \equiv e(t+T), \quad a>0 .
$$

Let us consider the auxiliary equation

$$
x^{\prime \prime \prime}+a x^{\prime \prime} \pm \lambda^{2} x=\mu\left\{e(t) \pm \lambda^{2} x-g(x)-f(x) x^{\prime}\right\}, \quad 0 \leqq \mu \leqq 1,
$$

which for $\mu=0$ is a linear one without non-trivial periodic solutions in case $\lambda>0$. As above, its $T$-periodic solutions can be represented as the continuous solutions of an integral equation of Hammerstein type. According to Leray-Schauder there is at least one solution of this equation for $\mu=1$ (that means, a periodic solution of (19)) if the solutions are a priori bounded for $0<\mu<1$.

Multiplying equation (20) formed for a $T$-periodic solution by this solution and integrating from 0 to $T$ we obtain the same result as in case (3) or (17) since

$$
x^{\prime \prime \prime} x=\frac{d}{d t}\left(x^{\prime \prime} x-\frac{1}{2} x^{\prime 2}\right)
$$

Thus, we can state 
THEOREM 4. - The third order equation (19) possesses at least one T-periodic solution if

$\left.a^{\prime}\right) x g(x) \geqq 0(|x| \geqq h>0), \quad \lim _{|x| \rightarrow \infty} \frac{g(x)}{a x}=k<\omega^{2}$,

$\left.b^{\prime}\right) \int_{0}^{T} e(t) d t=0$ in case $k=0$

$o r$

$$
0<\delta \leqq \frac{g(x)}{a x} \leqq k<\frac{\omega^{2}}{4}(|x| \geqq h)
$$

or

$$
\begin{aligned}
& \left.a^{\prime \prime \prime}\right) x g(x) \leqq 0 \quad(|x| \geqq h), \\
& \left.b^{\prime \prime \prime}\right) \int_{0}^{T} e(t) d t=0 .
\end{aligned}
$$

At last, we deal with the Rayleigh equation

$$
x^{\prime \prime}+F\left(x^{\prime}\right)+g(x)=e(t) \equiv e(t+T)
$$

$\left[F\left(x^{\prime}\right), g(x), e(t)\right.$ continuous functions $]$. We state

THEOREM 5. - If

$$
g(x)=k x+\gamma(x) \quad \text { with } \quad 0<k<\omega^{2}, \quad|\gamma(x)| \leqq P,
$$

there is at least one T-periodic solution of equation (21).

The proof is based on the auxiliary equation

$$
x^{\prime \prime}+k x=\mu\left\{e(t)-\gamma(x)-F\left(x^{\prime}\right)\right\}, \quad 0<\mu<1 ;
$$

it is shown that each $T$-periodic solution and its first derivative are a priori bounded. lution

Using, for brevity, the notation $(x, y)=\int_{0}^{T} x(t) y(t) d t$ we calculate for such a so-
ion

$$
\begin{aligned}
& \left\|x^{\prime \prime}\right\|^{2}+k\left(x, x^{\prime \prime}\right)=\mu\left(e(t)-\gamma(x), x^{\prime \prime}\right), \\
& \left\|x^{\prime \prime}\right\|^{2} \leqq k\left\|x^{\prime}\right\|^{2}+\sqrt{T}(E+P)\left\|x^{\prime \prime}\right\| .
\end{aligned}
$$

In the estimate (5) which requires only the mean convergence of the Fourier expansions we replace $x$ by $x^{\prime}$ and $x^{\prime}$ by $x^{\prime \prime}$; since $\int_{0}^{T} x^{\prime}(t) d t=0$ we obtain

$$
\left(1-\frac{k}{\omega^{2}}\right)\left\|x^{\prime \prime}\right\|^{2} \leqq \sqrt{ } T(E+P)\left\|o^{\prime \prime}\right\|
$$


that is,

$$
\left\|x^{n}\right\| \leqq x^{\prime \prime}(E+P)
$$

Let $\tau \in[0, T]$ be a zero of $x^{\prime}$; then we have

$$
\left|x^{\prime}(t)\right|=\left|\int_{\tau}^{t} x^{\prime \prime}(s) d s\right| \leqq \sqrt{T}\left\|x^{\prime \prime}\right\| \leqq x_{0}^{\prime}(E+P)=Y \quad \text { for } \tau \leqq t \leqq \tau+T .
$$

Integration of equation (22) from 0 to $T$ yields:

$$
\begin{aligned}
& k \int_{0}^{T} x(t) d t=\mu \int_{0}^{T}\left\{e(t)-\gamma(x(t))-F\left(x^{\prime}(t)\right)\right\} d t \\
& k: \int_{0}^{T} x(t) d t \mid \leqq(E+P+F) T \text { where } F=\operatorname{Max}_{|y| \leqq Y}|F(y)| .
\end{aligned}
$$

Let $x(\tau)=T^{-1} \int_{0}^{T} x(t) d t$ for some $\tau \in[0, T] ;$ then

$$
|x(t)| \leqq|x(\tau)|+\int_{\tau}^{t}\left|x^{\prime}(s)\right| d s \leqq \frac{E+P+F}{k}+T Y \text { for } \tau \leqq t \leqq \tau+T .
$$

The proof can be extended immediately to the third order equation

$$
x^{i \prime \prime}+a x^{\prime \prime}+F\left(x^{\prime}\right)+g(x)=e(t) \equiv e(t+T), \quad a \neq 0:
$$

If

$$
g(x)=a k x+\gamma(x), \quad 0<k<\omega^{2} \text { and }|\gamma(x)| \leqq P,
$$

then equation (23) admits at least one $T$-periodic solution.

\section{REFERENCES}

[1] T. A. BURToN - C. G. TOWNSEND, On the generalized tiénard equation with forcing term, J. Differential Equations, 4 (1968), pp. 620-633.

[2] R. FAJRE, Solutions périodiques d'équations différentielles et méthode de Leray-Schauder (Cas de vibrations forcées), Ann. Inst. Fourier (Grenoble), 14 (1964), pp. 195-204. 
[3] J. R. GRAEF, On the generalized Liénard equation with negative damping, J. Differential Equations, 12 (1972), pp. 34-62.

[4] D. Graffi, Sulle oscillazioni forzate nella meccanica non-lineare, Riv. Mat. Univ. Parma, 3 (1952), pp. 317-326.

[5] A. C. LAZER, On Schauder's fixed point theorem and forced second-order nonlinear oseillations, J. Math. Analysis Appl., 21 (1968), pp. 421-426.

[6] J. Mawhin, Degré topologique et solutions périodiques des systèmes différentiels non linéaires, Bull. Soc. Royale Sci. Liège, 38 (1969), pp. 308-398.

[7] J. MAWHIN, An extension of a theorem of A. C. Lazer on forced nonlinear oscillations, J. Math. Analysis Appl., 40 (1972) pp. 20-29.

[8] R. Reissig - G. Sansone - R. Conti, Nichtlineare Differentialgleiohungen höherer Ordmung, Roma, 1969.

[9] G. SANSONE - R. Conti, Nonlinear differential equations (translated from the Italian by A. H. Diamond), Oxford, 1964. 